\title{
Brain Morphometry by Probabilistic Latent Semantic Analysis
}

\author{
U. Castellani ${ }^{1}$, A. Perina ${ }^{1}$, V. Murino ${ }^{1,2}$, M. Bellani ${ }^{2}$, G. Rambaldelli ${ }^{3}$, \\ M. Tansella ${ }^{3}$, and P. Brambilla ${ }^{3,4}$ \\ ${ }^{1}$ VIPS lab, University of Verona, Italy \\ ${ }^{2}$ Istituto Italiano di Tecnologia (IIT), Italy \\ ${ }^{3}$ Department of Medicine and Public Health, University of Verona, Italy \\ ${ }^{4}$ ICBN Center, University of Udine and Verona, Italy
}

\begin{abstract}
The paper proposes a new shape morphometry approach that combines advanced classification techniques with geometric features to identify morphological abnormalities on the brain surface. Our aim is to improve the classification accuracy in distinguishing between normal subjects and schizophrenic patients. The approach is inspired by natural language processing. Local brain surface geometric patterns are quantized to visual words, and their co-occurrences are encoded as visual topic. To do this, a generative model, the probabilistic Latent Semantic Analysis is learned from quantized shape descriptors (visual words). Finally, we extract from the learned models a generative score, that is used as input of a Support Vector Machine (SVM), defining an hybrid generative/discriminative classification algorithm. An exhaustive experimental section is proposed on a dataset consisting of MRI scans from 64 patients and 60 control subjects. Promising results are reporting by observing accuracies up to $86.13 \%$.
\end{abstract}

\section{Introduction}

Computational neuroanatomy using magnetic resonance imaging (MRI) is a fruitful research field that employs image processing techniques to identify geometric characteristics of different brains [1]. The ultimate goal is to automatically identify structural brain abnormalities by estimating the differences between normal subjects and patients affected by a certain disease. Standard approaches are based on the measurement of volume variations [2] which are useful to explain atrophy or dilation due to illness, but on the other hand, they lack in detecting small structural abnormalities at specific locations. To this aim, more advanced shape analysis techniques have been proposed 3/4,5]. In [3] geometric properties are captured by computing spherical harmonic descriptors on brain surfaces. Although results are effective, the method requires shapes registration and data resampling. Such pre-processing is avoided in [45], where the so called Shape-DNA signature has been introduced by taking the eigenvalues of the Laplace-Beltrami operator as region descriptor for both external surface and volume. Moreover, possible morphological anomalies can be localized by the 
analysis of the eigenfunctions. Recently a more general shape analysis technique has been proposed, namely the feature-based morphometry (FBM) [6]. FBM identifies anatomical structure that can be used as disease biomarkers without a one-to-one spatial correspondence between all subjects. In order to improve the capability in distinguishing between healthy and non-healthy subjects, learning by example techniques [7] are applied (see for example, 8]). Usually, geometric signatures extracted from the MRI data are used as feature vector for classification purpose 91011]. In [9] a support vector machine (SVM) has been employed to classify cortical thickness which has been measured by calculating the Euclidean distance between linked vertices on the inner and outer cortical surfaces. In 10, a new approach has been defined by combining deformation-based morphometry with SVM. In this fashion, multivariate relationships among various anatomical regions have been captured to characterize more effectively the group differences. Finally, in [11, a unified framework is proposed to combine advanced probabilistic registration techniques with SVM. The local spatial warps parameters are also used to identify the discriminative warp that best differentiates the two groups.

In this paper we proposed a new shape morphometry approach. We combine geometric surface properties extraction with advanced classification techniques. Firstly, local geometric features are computed from the brain surface to characterize single surface vertices. Then, being inspired by the research on natural language processing, geometric features are quantized into local shape configurations by defining the set of visual words. In order to improve the shape description, a generative model is learned to find local patterns of co-occurrences, by leading to the definition of the so called visual topics. To this aim we employ a generative model, the probabilistic Latent Semantic Analysis (pLSA [12]). Finally, the topic distributions of each subject are fed to a SVM classifier by defining a proper generative kernel. In this fashion the classifier employs a discriminative-generative approach which represents one of the most effective and advanced classification paradigms. In this work, we focus on schizophrenia by analyzing a dataset of 64 patients and 60 controls. A Region-of-Interest (ROI)-based approach is employed by studying the left-Amygdala. The proposed method is able to satisfy at the same time the main advantages of the previous methods: i) registration is not required as in 456, since extracted geometric features are position and scale invariant, ii) morphometric abnormalities can be localized and visualized as in [311, iii) promising accuracy classification performances are observed by exploiting advanced classification techniques, as suggested in [8]. Moreover, the idea of encoding the geometric pattern cooccurrences as topics of a generative model is new for shape analysis and for the biomedical domain, for the best of our knowledge.

\section{Geometric Feature Extraction}

From the set of 2D ROIs of the left-Amygdala shapes the 3D surface is computed as triangle mesh using marching cubes. A minimal smoothing operation is 
applied to remove noise and voxelization effect. We encode geometric properties of the surface using the Shape Index [13, which is defined as:

$$
s i=-\frac{2}{\pi} \arctan \left(\frac{k_{1}+k_{2}}{k_{1}-k_{2}}\right) \quad k_{1}>k_{2},
$$

where $k_{1}, k_{2}$ are the principal curvatures of a generic surface point. The Shape Index varies in $[-1,1]$ and provides a local categorization of the shape into primitive forms such as spherical cap and cup, rut, ridge, trough, or saddle 13. Shape index is pose and scale invariant [13] and it has already been successfully employed in biomedical domain 14. The shape index is computed at each vertex of the extracted mesh. Then, all the values are quantized and an histogram of occurrences is computed. Such histogram represent the descriptor of a given subject and it basically encodes the brain local geometry of a subject, disregarding the spatial relationships. Figure 1 shows the 3D surface of the left-Amygdala
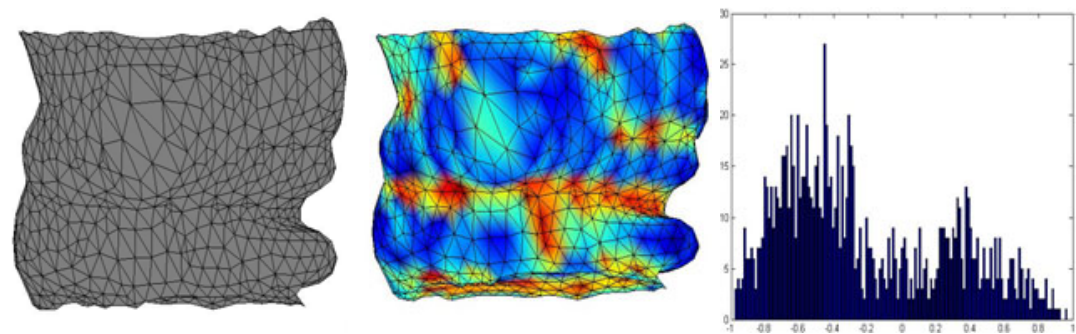

Fig. 1. Geometric feature extraction: 3D surface of the left-Amygdala (left), the surface colored according with Shape Index values (center), and the histogram of Shape Index occurrences (right)

(left), the surface colored according with Shape Index values (center), and the histogram of Shape Index occurrences (right). It is worth noting that convex regions (in blue) are clearly distinguished from concave regions (in red) by the Shape Index values.

\section{$3 \quad$ Topic Models and pLSA}

Topic models were introduced in the linguistic scenario, to describe and model documents. The basic idea underlying these methods is that each document is seen as mixture of topics - where a topic is a probability distribution over words. Intuitively, each topic may be related to a particular set of co-occurrent words, that are specific of the treated argument. This representation has one clear advantage: each topic is individually interpretable, providing a probability distribution over words that picks out a coherent cluster of correlated terms. In the shape analysis context, this can be really advantageous since the final goal is to 
provide knowledge about morphological abnormalities, and infer possible hidden correlations. In this paper, we focus on probabilistic Latent Semantic Analysis (pLSA) 12 which takes as input a dataset of $N$ documents $\left\{d_{i}\right\}, i=1, \ldots, N$, encoded by set of words. Before applying pLSA, the dataset is summarized by a co-occurrence matrix of size $M \times N$, where the entry $n\left(w_{j}, d_{i}\right)$ indicates the number of occurrences of the word $w_{j}$ in the document $d_{i}$. The presence of a word $w_{j}$ in the document $d_{i}$ is mediated by a latent topic variable, $z \in T=\left\{z_{1}, \ldots, z_{Z}\right\}$, also called aspect class, i.e.,

$$
P\left(w_{j}, d_{i}\right)=\sum_{k=1}^{Z} P\left(w_{j} \mid z_{k}\right) P\left(z_{k} \mid d_{i}\right) P\left(d_{i}\right) .
$$

In practice, the topic $z_{k}$ is a probabilistic co-occurrence of words encoded by the distribution $P\left(w \mid z_{k}\right), w=\left\{w_{1}, \ldots, w_{M}\right\}$, and each document $d_{i}$ is compactly $(Z<M)^{1}$ modeled as a probability distribution over the topics, i.e., $P\left(z \mid d_{i}\right)$, $z=\left\{z_{1}, \ldots, z_{Z}\right\} ; P\left(d_{i}\right)$ accounts for varying number of words. The hidden distributions of the model, $P(w \mid z)$ and $P(z \mid d)$, are learnt using Expectation-Maximization (EM), maximizing the model data-likelihood L:

$$
L=\prod_{i=1}^{N} \prod_{j=1}^{M} P\left(w_{j}, d_{i}\right)^{n\left(w_{j}, d_{i}\right)}
$$

The E-step computes the posterior over the topics, $P(z \mid w, d)$, and the M-step updates the parameters, $P^{C}(w \mid z)$ which identifies the model. Once the model has been learnt, the goal of inference is to estimate the topic distribution of a novel document. To do this, one can use the standard learning algorithm keeping fixed the parameters $P(w \mid z)$.

To perform generative classification with pLSA one has to learn a model perclass and assign a new sample to the category whose model fits the point best, i.e., the model with highest likelihood (see Equation 2). Recently, other approaches successfully used meaningful distributions or other by-products coming from a generative model, as feature for a discriminative classifier. The feature vector resulting after this mapping is called generative score. The intuition is that generative models like pLSA are built to understand how samples were generated, and they haven't any notion of discrimination; on the other hand, discriminative classifiers are built to separate the data and they are highly more effective if the data has been previously "explained" by a generative model.

Here turn now to explain how we adopt this ideas to the problem in hand: in our case the words $w_{j}$ are the quantized geometric features previously presented and the documents $d$ are the subjects. The number of bins (words) with which we quantize the continuous shape index values $(\mathrm{M}=150)$ has been heuristically chosen in order to get the best possible performance by classifying directly the feature histograms. As result, the input of pLSA are histograms of quantized shape index values and we want to look for co-occurrence of geometric features.

${ }^{1}$ Both $Z$ and $M$ are constants to be a-priori set. 
To classify, we learn a pLSA model for normal controls (i.e., $P^{C}(w \mid z)$ ) and one for schizophrenic patients (i.e., $P^{S}(w \mid z)$ ). Instead of comparing the likelihoods of the two models to classify, we extract a score $\phi(d)$ for each document $d$ according its estimated pLSA posteriors distribution, to use it with a discriminative classifier. In forumlae we have that

$$
\phi(d)=\left[P\left(z^{C} \mid d\right), P\left(z^{S} \mid d\right)\right]=\left[P\left(z_{1}^{C} \mid d\right), \cdots P\left(z_{Z}^{C} \mid d\right), P\left(z_{1}^{S} \mid d\right), \cdots P\left(z_{Z}^{S} \mid d\right)\right]
$$

Our intuition is that the co-occurrence of geometric features is different between controls and cases. Since the co-occurrences are captured by the topic distributions $P\left(z^{C} \mid d\right)$ and $P\left(z^{S} \mid d\right)$, we are defining a meaningful score for discrimination.

\section{SVM Classification}

One of the most powerful classifier for object recognition is the Support Vector Machine (SVM). SVM constructs a maximal margin hyperplane in a high dimensional feature space, by mapping the original features through a kernel function. Here, the input of the SVM are the score vectors (Eq. 3). In order to compare data, we employed two kind of kernels: the Histogram Intersection (HI) kernel and the $\chi^{2}$-kernel. Given two brains $A$ and $B$, described by their quantized shape index distributions $d^{A}$ and $d^{B}$, HI and $\chi^{2}$ kernel functions are respectively defined as:

$$
K_{H I}(A, B)=\sum_{i=1}^{2 Z} \min \left[\phi_{i}\left(d^{A}\right), \phi_{i}\left(d^{B}\right)\right] ; \quad K_{\chi^{2}}(A, B)=\sum_{i=1}^{2 Z} \min \left[\phi_{i}\left(d^{A}\right), \phi_{i}\left(d^{B}\right)\right]
$$

where $\phi_{i}(d)=P\left(z_{i} \mid d\right)$, and $2 \cdot \mathrm{Z}$ is the length of the score vector.

In this fashion, according to the generative/discriminative classification paradigm, we use the information coming from the generative process as discriminative features of a discriminative classifier. In practice, we measure similarities in the employed topics during the generation of a sample.

\section{Results}

The dataset used in this work is composed by 64 patients affected by schizophrenia and 60 healthy control subjects, making our dataset much larger than those commonly used in schizophrenia.

MRI scans were acquired using a 1.5 T Siemens Magnetom Symphony Maestro Class, Syngo MR 2002B by facing several methodological issues dictated by international organization to minimize biases and distortions.

We focused our analysis on the left-Amygdala whose abnormal activity is already investigated in schizophrenia. Regions have been manually traced by experts, according to well established medical guidelines. After the geometric feature extraction and pLSA analysis, two generative models are available: one for normal controls and one for schizophrenic patients. 

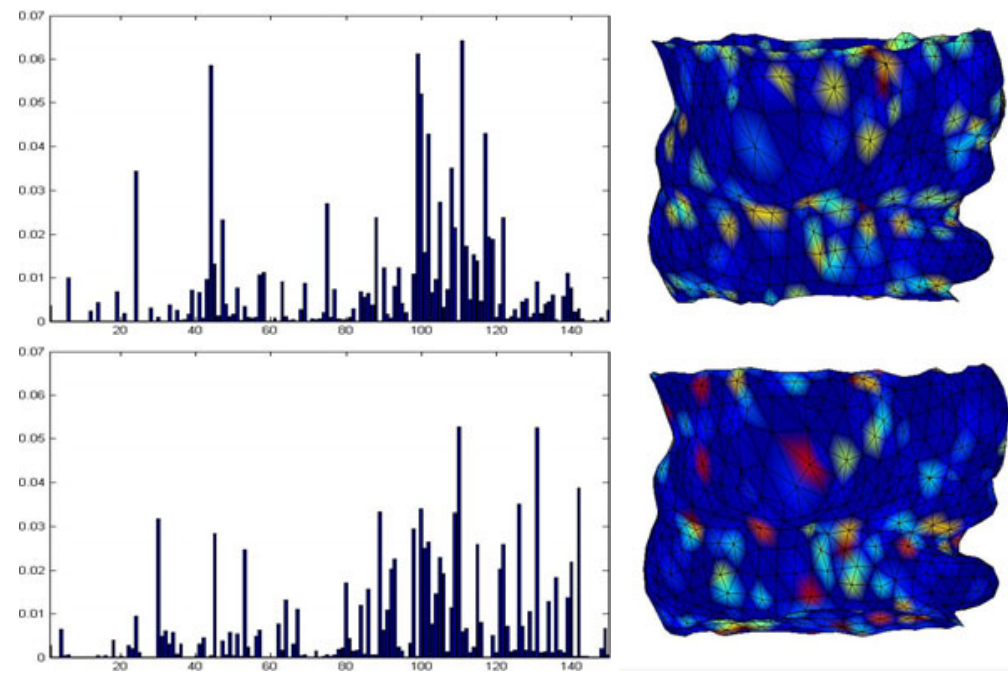

Fig. 2. Output of pLSA: best-topic for healthy-model $P^{C}\left(w \mid z=\hat{z_{c}}\right)$ (top) and patientmodel $P^{S}\left(w \mid z=\hat{z_{s}}\right)$ (bottom). Visual words distribution (left) and their projection onto the surface (right).

In order to obtain a visual feedback of the generative process, we select one high discriminative topic per class, $\hat{z_{c}}$ and $\hat{z_{s}}$, respectively for controls and schizophrenic patients. In Figure 2 (left) the word-distribution for both topics is plotted (i.e., the best-topic of the healthy model on the top, and of the patient model on the bottom). Note how the visual words are differently generated by the two topics. Moreover, in Figure 2 (right) we show the brain surface of a patient by coloring each vertex according to the relevance of its respective words for the best-topic of the healthy model (top) and the patient model (bottom). In this fashion is possible to highlight the surface areas which are more related to the disease, in a likelihood sense (and vice versa for healthy areas).

The classification stage is employed by a random cross-validation strategy. We randomly extracted the $75 \%$ of the samples as training set, using the rest for testing. The process is repeated 20 times. Moreover, we investigated the effect of the performance by varying the number of topics $Z$ necessary to capture all the co-occurrences.

Results are reported in Figure 3 a. Our best result is $86.13 \% \pm 2.17$ obtained with 45 topics and histogram intersection kernel. This is impressive if compared with the SVM classification performed directly on the feature histograms $n(w, d)$ by using the same validation strategy and kernel (mean accuracy $58.70 \% \pm 9.78$ ) and it shows how beneficial is the proposed generative modeling part.

Although an optimal value of $Z$ should be automatically estimated, our method is robust to the choice of $Z$. Indeed, we show satisfactory results, i.e., higher than $80 \%$ for each $Z \in[25,55]$. 
a) Proposed Approach (pLSA reduction)
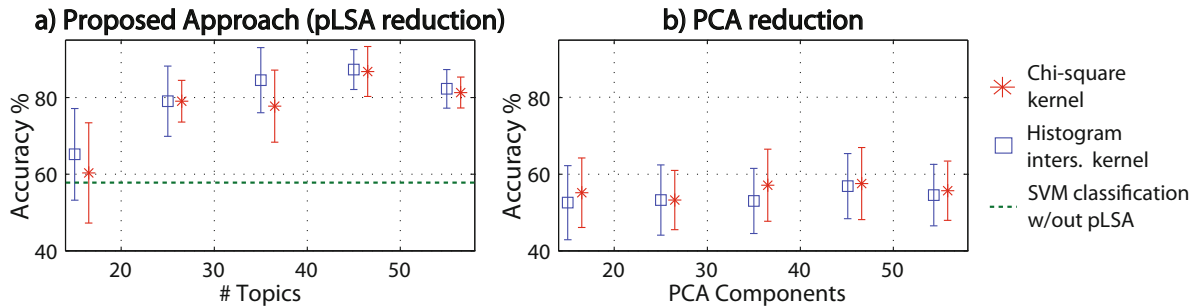

Fig. 3. Error bars showing mean accuracy and standard deviation obtained by varying the number of topics $Z$. a) The proposed approach. b) PCA dimensionality reduction. Please note how best classification accuracies have lower standard deviations.

As further test, we reduced the dimensionality of the quantized shape index histograms $d^{A}$ with principal component analysis (PCA), varying the saved components $K \in[25,55]$. Then, we classified with the same kernels we employed for the proposed approach. Results for PCA, shown in Figure 3b, slightly exceed the chance and they demonstrate how pLSA-based dimensionality reduction is much more discriminative than the one obtained with PCA.

Finally, it is worth noting that our results are in accordance with other similar methods. For instance [11] has shown an accuracy of $90.00 \%$ in classifying between 16 first episode schizophrenic and 17 age-matched healthy subjects. In [10], results showed $91.8 \%$ of accuracy between 23 cases and 38 controls (only female). Our performance is slightly less but we are analyzing a wider database (no age-sex distinctions).

\section{Conclusions}

In this paper a new approach for shape analysis is proposed with the aim at characterizing morphological features in schizophrenia. Surface geometric properties are carefully modeled by focusing on left-Amigdala. We have shown that a drastic improvement is observed when the co-occurrences of geometric features are taking into account for classification. In particular, from the pLSA analysis is possible to evaluate the relevance of topics and visual words in modeling both the populations (i.e., healthy and an-healthy people). This open new investigative perspectives in localizing abnormalities due to the presence of multiple geometric factors at the same time. With our method, we have obtained satisfactory results in classification accuracy (i.e., up to $86.13 \%$ ) which are coherent with previous studies in schizophrenia. Moreover, we have analyzed a larger number subjects in comparison with related work by improving the statistical relevance of the proposed experiments.

\section{Acknowledgements}

We acknowledge financial support from the FET programme within the EU-FP7, under the SIMBAD project (contract 213250). 


\section{References}

1. Giuliani, N.R., Calhouna, V.D., Pearlson, G.D., Francis, A., Buchanan, R.W.: Voxel-based morphometry versus region of interest: a comparison of two methods for analyzing gray matter differences in schizophrenia. Schizophrenia Research 74(2-3), 135-147 (2005)

2. Shenton, M.E., Dickey, C.C., Frumin, M., McCarley, R.W.: A review of mri findings in schizophrenia. Schizophrenia Research 49(1-2), 1-52 (2001)

3. Styner, M., Oguz, I., Xu, S., Brechbuhler, C., Pantazis, D., Levitt, J., Shenton, M., Gerig, G.: Framework for the statistical shape analysis of brain structures using spharm-pdm. In: Open Science Workshop at MICCAI (2006)

4. Niethammer, M., Reuter, M., Wolter, F.E., Bouix, S., Peinecke, N., Koo, M.S., Shenton, M.: Global medical shape analysis using the laplace-beltrami spectrum. In: Ayache, N., Ourselin, S., Maeder, A. (eds.) MICCAI 2007, Part I. LNCS, vol. 4791, pp. 850-857. Springer, Heidelberg (2007)

5. Reuter, M., Wolter, F.E., Shenton, M., Niethammer, M.: Laplace-Beltrami eigenvalues and topological features on eigenfuntions for statistical shape analysis. Computed-Aided Design 41(10), 739-755 (2009)

6. Toews, M., Wells III, W.M., Collins, D.L., Arbel, T.: Feature-based morphometry. In: Yang, G.-Z., Hawkes, D., Rueckert, D., Noble, A., Taylor, C. (eds.) MICCAI 2009. LNCS, vol. 5762, pp. 109-116. Springer, Heidelberg (2009)

7. Duda, R., Hart, P., Stork, D.: Pattern Classification, 2nd edn. John Wiley \& Sons, Chichester (2001)

8. Davatzikos, C.: Why voxel-based morphometric analysis should be used with great caution when characterizing group differences. NeuroImage 23(1), 17-20 (2004)

9. Yoon, U., Lee, J., Im, K., Shin, W., Cho, B.H., Kim, I., Kwon, J., Kim, S.: Pattern classification using principal components of cortical thickness and its discriminative pattern in schizophrenia. NeuroImage 34, 1405-1415 (2007)

10. Fan, Y., Shen, D., Gur, R.C., Gur, R.E., Davatzikos, C.: Compare: Classification of morphological patterns using adaptive regional elements. IEEE Transactions on Medical Imaging 26(1), 93-105 (2007)

11. Pohl, K.M., Sabuncu, M.R.: A unified framework for $\mathrm{mr}$ based disease classification. In: Prince, J.L., Pham, D.L., Myers, K.J. (eds.) IPMI 2009. LNCS, vol. 5636, pp. 300-313. Springer, Heidelberg (2009)

12. Hofmann, T.: Unsupervised learning by probabilistic latent semantic analysis. Mach. Learn. 42(1-2), 177-196 (2001)

13. Koenderink, J., van Doorn, A.: Surface shape and curvature scales. Image and Visual Computing 10, 557-565 (1992)

14. Awate, S.P., Yushkevich, P., Song, Z., Licht, D., Gee, J.C.: Multivariate highdimensional cortical folding analysis, combining complexity and shape, in neonates with congenital heart disease. In: IPMI (2009) 